\title{
Why brown fat has a lot of nerve
}

Brown fat in mammals burns stored calories by producing heat, in a process that is controlled by nerves infiltrating this tissue. A protein that regulates innervation of brown fat and heat generation has been discovered. SEE ARTICLE P.229

\section{REJJI KURUVILLA}

$\mathrm{T}$ The dramatic rise in obesity has led to a global increase in diabetes and cardiovascular disease. Excess calories in mammals are stored as triglyceride molecules in white adipose tissue (also known as white fat). By contrast, brown adipose tissue (BAT; brown fat) burns fat and glucose molecules to generate heat in a process called adaptive non-shivering thermogenesis ${ }^{1}$. Activating BAT thermogenesis is a potential strategy for combating obesity, so there is great interest in understanding the development and physiology of this tissue. Zeng et al. ${ }^{2}$ report on page 229 that the protein calsyntenin $3 \beta$ (CLSTN3 $\beta$ ), found in brown fat cells, acts as a crucial regulator of BAT innervation and thermogenesis by controlling the secretion of a growth factor, the protein S100b.

A key regulator of BAT thermogenesis is the sympathetic nervous system ${ }^{3}$, which mediates neuronal and hormonal responses to stress - better known as the fight-or-flight response. Sympathetic nerves innervate BAT much more abundantly than they do white fat, and release noradrenaline molecules that bind to and activate adrenergic receptors on BAT heat-producing cells (brown adipocytes; Fig. 1). Active adrenergic receptors produce heat by triggering biochemical events in mitochondria - the cell's energy-generating organelles - with the help of a specialized mitochondrial protein, known as uncoupling protein 1 (UCP1).

Zeng et al. set out to investigate the processes that control the innervation of BAT. By analysing all the RNA transcripts produced in brown adipocytes, the authors identified a previously uncharacterized, mammalianspecific gene - Clstn $3 b$ - that is highly and selectively expressed in these cells. They observed that the expression of Clstn $3 b$ is also induced in beige fat by exposure to cold; beige fat consists of adipocytes in white fat that can be triggered to become thermogenic by cold and other stimuli.

Strikingly, when the researchers genetically engineered 'knockout' mice that lack Clstn $3 b$, the animals rapidly developed hypothermia in response to acute cold, which is indicative of defective thermogenesis. These mice were also heavier than their wild-type counterparts, had

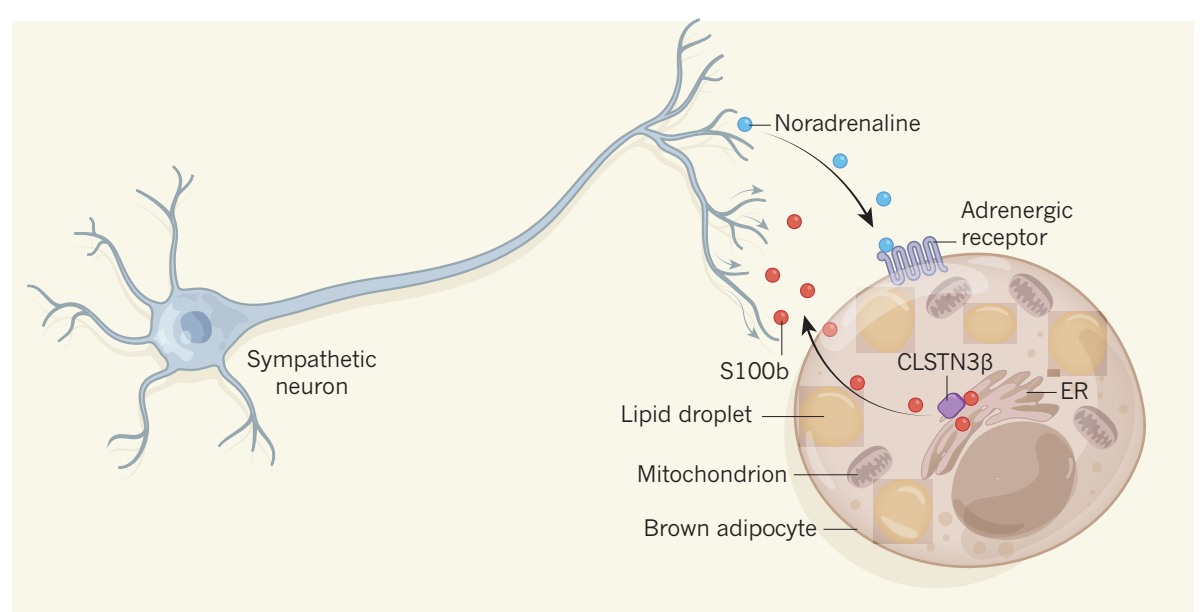

Figure 1 | The calsyntenin $3 \beta$ protein regulates innervation of brown fat. Brown fat is highly infiltrated by nerves of the sympathetic nervous system (the part of the nervous system that controls the flight-or-fight response). Sympathetic neurons release noradrenaline molecules that bind to and activate adrenergic receptors on brown fat cells (brown adipocytes). This activation triggers biochemical events (not shown) in mitochondria, the cell's energy-producing organelles, causing the adipocytes to burn calories stored as lipids, which generates heat. Zeng et al. ${ }^{2}$ have identified a protein called calsyntenin $3 \beta$ (CLSTN3 $\beta$ ) that is expressed in the endoplasmic reticulum (ER, an intracellular organelle) of brown adipocytes. They report that calsyntenin $3 \beta$ aids the secretion of a growth-factor protein, S100b, from brown adipocytes, and that $\mathrm{S} 100 \mathrm{~b}$ facilitates the growth of projections from neurons.

increased fat deposits and decreased oxidative activity in brown adipocytes, and became obese and had raised levels of blood glucose (glucose intolerance) on a high-fat diet. Conversely, genetically engineered mice that overexpress Clstn $3 b$ in brown adipocytes had lower body weights than wild-type mice and improved cold tolerance, and were resistant to diet-induced obesity and glucose intolerance. Together, these studies indicate that Clstn3b is necessary and sufficient for BAT thermogenesis and whole-body energy expenditure.

How does calsyntenin $3 \beta$ control BAT function? Despite the altered cold sensitivity of the Clstn3b-knockout mice, the animals' isolated brown adipocytes showed normal respiratory responses (oxygen consumption in mitochondria) when treated acutely with noradrenaline. Similar results were obtained at the whole-body level when the knockout mice were treated with an adrenergic-receptor activator, indicating that the noradrenalineresponsive machinery is intact in these animals. However, Zeng et al. observed that sympathetic innervation in BAT is affected by the level of expression of Clstn $3 b$ : compared with wild-type mice, there was a decrease in the density of sympathetic nerve fibres in Clstn3b-knockout mice, but an increase in Clstn3b-overexpressing mice.

The authors observed that calsyntenin $3 \beta$ is found in the membrane of the endoplasmic reticulum (ER) of brown adipocytes; the ER is an intracellular organelle in which many secreted and membrane proteins are synthesized. So how does it influence BAT innervation? The researchers found that the protein that is most strongly downregulated in the BAT of Clstn $3 b$-knockout mice is S100b, which is abundant in brain cells called astrocytes ${ }^{5}$. Intriguingly, Zeng et al. find that calsyntenin $3 \beta$ interacts physically with $S 100 \mathrm{~b}$, and thereby acts as a chaperone protein that directs and aids the secretion of newly formed S100b from the ER.

The authors found that when they treated cultured sympathetic neurons with soluble S100b, extension of nerve projections was promoted. Moreover, BAT innervation in mice lacking S100b was reduced compared with that in wild-type mice, whereas innervation of other organs, such as the salivary glands, was 
unaffected. Remarkably, forced expression of S100b in the brown adipocytes of Clstn3bknockout mice is sufficient to correct the deficits in sympathetic innervation and thermogenesis in these animals. The emerging picture is of an adipocyte-derived mechanism that mediates crosstalk between brown adipocytes and innervating sympathetic fibres (Fig. 1).

Zeng and colleagues' findings raise several questions. For example, how is calsyntenin $3 \beta$ expression regulated in brown adipocytes? The authors found that one upstream regulator of this protein is the enzyme lysine-specific demethylase 1 (LSD1), which mediates the differentiation of brown and beige fat. Adipose-specific loss of LSD1 in mice causes downregulation of BAT-specific genes and aberrant induction of genes typically found in white adipose tissue ${ }^{6}$.

The growth of sympathetic neurons mostly relies on a protein called nerve growth factor (NGF). NGF-deficient mice have pronounced disruption of sympathetic innervation of multiple peripheral tissues ${ }^{7}$, but BAT innervation has not been specifically examined in this context. It remains to be seen whether NGF acts together with S100b to coordinate BAT innervation, or is dispensable, as in the innervation of the trachea ${ }^{7}$. Could other factors derived from peripheral tissue control the sympathetic innervation of specific targets? If so, they could be useful tools for manipulating such innervation and studying its role in individual tissues.

Sympathetic innervation controls the expression of genes involved in thermogenesis and the differentiation of brown and beige fat ${ }^{4}$. However, Zeng et al. found that loss of $C l \sin 3 b$ does not affect the expression of genes involved in adipocyte development, thermogenesis or mitochondrial function, including the gene that encodes UCP1. Only the expression of the Dio2 gene, which encodes an enzyme that produces the active form of a thyroid hormone, is significantly altered by the loss or overexpression of Clstn $3 b$. The Dio2 protein is abundant in BAT, and, as is seen in the Clstn3b-knockout mice, mice lacking Dio2 have impaired thermogenesis and decreased cold tolerance, and are susceptible to dietinduced obesity ${ }^{8}$. Altered thyroid signalling might therefore contribute to BAT dysfunction in the absence of calsyntenin $3 \beta$.

The final issue arising from Zeng and colleagues' work is how calsyntenin $3 \beta$ promotes the secretion of S100b. The synthesis of proteins destined for cellular secretion is initiated in the cytoplasm. The nascent proteins typically contain an amino-acid sequence known as a signal peptide, which directs them from the cytoplasm to the ER for the start of their secretory journey while they are still being synthesized. However, some secreted proteins lack signal peptides and are imported to the ER after they have been synthesized ${ }^{9}$. S100b lacks a peptide signal, and so the new findings raise the question of whether calsyntenin $3 \beta$ functions as a general ER chaperone for the secretion of proteins that lack signal peptides, or is specific for S100b.

In humans, BAT had been thought to exist only in infants, until imaging studies revealed deposits of thermogenic brown fat in adults ${ }^{10}$. Clstn $3 b$ is expressed in human adipose tissue, and might be involved in the regulation of BAT innervation, as it is in mice. Zeng and colleagues' study might therefore inform therapeutic strategies to enhance sympathetic innervation, and thereby harness the thermogenic potential of BAT to combat obesity and its metabolic consequences.

Rejji Kuruvilla is in the Department of Biology, Johns Hopkins University, Baltimore,
Maryland 21218, USA.

e-mail: rkuruvilla@jhu.edu

1. Cannon, B. \& Nedergaard, J. Physiol. Rev. 84, 277-359 (2004).

2. Zeng, X. et al. Nature 569, 229-235 (2019).

3. Bartness, T. J., Vaughan, C. H. \& Song, C. K. Int. J. Obesity 34, S36-S42 (2010).

4. Wang, W. \& Seale, P. Nature Rev. Mol. Cell Biol. 17 , 691-702 (2016).

5. Ludwin, S. K., Kosek, J. C. \& Eng, L. F. J. Comp. Neurol. 165, 197-207 (1976).

6. Duteil, D. et al. Cell Rep. 17, 1008-1021 (2016).

7. Glebova, N. O. \& Ginty, D. D. J. Neurosci. 24 743-751 (2004).

8. Hall, J. A. etal. Endocrinology 151, 4573-4582 (2010).

9. Rabouille, C. Trends Cell Biol. 27, 230-240 (2017).

10. Nedergaard, J., Bengtsson, T. \& Cannon, B. Am. J. Physiol. Endocrinol. Metab. 293, E444-E452 (2007).

This article was published online on 1 May 2019.

\section{TRANSCRIPTION}

\section{Gene-expression maps fall into place}

\section{Knowing the gene-expression pattern of individual cells can unlock their identity. A refined method for generating cellular RNA profiles offers a way to obtain such data at a high level of spatial resolution in intact tissues.}

\section{SAMANTHA A. MORRIS}

$\mathrm{M}$ onitoring messenger RNA in cells is a way to gather data that provides many biological insights. Improvements in the methods for detecting cellular mRNA are helping to provide more-detailed pictures of gene expression in the cells of a particular tissue. Writing in Science, Rodriques et al. ${ }^{1}$ describe a new approach to assessing cellular RNA that they term Slide-seq. This versatile technique couples high-throughput RNA sequencing with a way to capture spatial information about the location of the analysed cells in tissues.

Scientists often try to tackle the complexity of a biological system by breaking it down to focus on cells, and cataloguing these individual units on the basis of their identity. Conventional microscopic imaging of cells in a tissue can provide much information, such as cell type and function, but imaging data of many cells can still lack a high level of detail about cellular features - the characteristics known as the cellular phenotype.

The development of techniques for rigorously analysing the molecular contents of individual cells offers an alternative way to capture thousands of cellular features and generate an unbiased picture of the cells in a given tissue. For example, single-cell RNA sequencing has been rapidly and widely adopted since its emergence ten years ago ${ }^{2}$. The use of microfluidic-based technologies in this approach has brought huge efficiency gains and cost reductions $^{3-5}$. However, single-cell RNA sequencing requires tissue disruption and cell destruction, which causes loss of spatial information about cellular location that would be valuable for cell-type identification.

Efforts to retain spatial information are a current focus in the development of methods for single-cell analysis. For example, a method called multiplexed in situ hybridization, along with certain sequencing techniques, has enabled RNA to be monitored on a subcellular scale in intact tissues ${ }^{6-8}$. However, these approaches require substantial technical expertise, which has limited their widespread adoption.

Rodriques and colleagues' approach is conceptually rooted in an earlier ${ }^{3}$ method for single-cell RNA analysis. In that technique, called Drop-seq, the tissue being analysed is disrupted to separate the cells, which are then loaded onto a microfluidic device. Individual cells are encapsulated in nanolitrescale droplets together with a microbead coated with copies of a DNA sequence that provides a unique 'barcode' for the identification of material from each droplet (which is assumed to contain a single cell). The cells are broken open, which allows the cellular RNA to bind to the DNA barcodes. A step called reverse transcription generates DNA corresponding to the captured RNA sequences, and this DNA is tagged with the barcode DNA. DNA sequencing then enables 